\title{
Cinco años de abstinencia de todo ácido: La experiencia del grabado no tóxico en la Escuela de Artes Plásticas de la Universidad de Costa Rica
}

\section{Five years of total abstinence of any acid: University of Costa Rica's School of Fine Arts Non-Toxic printmaking experience}

\author{
Francisco Hernández-ChaVARría \\ Universidad de Costa Rica. Escuela de Artes Plásticas \\ franciscohernandezch@gmail.com \\ Alberto Murillo \\ Universidad de Costa Rica. Escuela de Artes Plásticas \\ albertomur@gmail.com
}

Recibido: 1 de noviembre de 2011

Aprobado: 19 de diciembre de 2011

\section{Resumen}

En los últimos cinco años el taller de grabado de la Escuela de Artes Plásticas de la Universidad de Costa Rica, se convirtió en un laboratorio de experimentación para probar cualquier idea en busca de erradicar el ácido nítrico y otras sustancias peligrosas, que tradicionalmente se han usado en la rutina diaria del grabado. Hemos introducido el uso de mordentes como el sulfato salino para grabar placas de hierro o aluminio, o el cloruro férrico para trabajar las placas de cobre. Además, hemos recuperado la electrólisis para grabar cualquiera de los metales mencionados. Actualmente, estos procesos son parte de nuestra rutina diaria, porque el ácido nítrico fue erradicado. Mientras tanto, se continúa la investigación de nuevas alternativas, más económicas y prácticas. Otra de nuestras preocupaciones es el uso de solventes de petróleo como rutina para limpiar cualquier cosa en el taller de grabado, y como respuesta a ese problema, sugerimos emplear aceites vegetales o limpiadores domésticos a base de limoneno. En conclusión, el último quinquenio ha sido exitoso en nuestro objetivo por erradicar cualquier sustancia o proceso peligroso del taller de grabado y continuamos haciendo esfuerzos para divulgar las ventajas de la metodología del grabado no tóxico.

Palabras Clave: Grabado no tóxico, electrolisis, sacrificio metálico, sulfato salino, cloruro férrico.

Hernández-Chavarría, F. Murillo, A. (2012): Cinco años de abstinencia de todo ácido: La experiencia del grabado no tóxico en la Escuela de Artes Plásticas de la Universidad de Costa Rica. Arte, Individuo y Sociedad, 24 (2) 167-177 


\begin{abstract}
In the last five years, the printmaking workshop at the University of Costa Rica's Fine Arts School has become a research laboratory, to probe any idea to eradicate the nitric acid and other harmful substances that has been used traditionally as normal in daily printmaking routine. We have introduced the use of saline etching solutions, such as saline-sulfate in etching iron, aluminum or zinc plates, and ferric chloride to erode copper plates. Furthermore, we have brought back electrolysis to etch any of the mentioned metals. Nowadays, these procedures are our every day work as the standard routine, because the nitric acid has been eradicated. Meanwhile, new alternatives, more economical and practical, are still been researched. Another cause of concern, it is the use of petroleum base solvents as a routine for cleaning everything in the printmaking workshop, and to answering that problem we suggest the use of vegetal oils or domestic cleaning products with limonene. In conclusions, the past five year period has been fruitful in our aim to eradicate any harmful substance or procedures from the printmaking workshop and, that we have been doing and continue to do efforts to divulgate the advantages of printmaking nontoxic methodologies.
\end{abstract}

Key Words: Non-toxic, electrolysis, metal sacrifice, saline sulfate, iron chloride.

Hernández-Chavarría, F. Murillo, A. (2012): Five years of totally abstinence of any acid: University of Costa Rica's School of Fine Arts Non-Toxic printmaking experience. Arte, Individuo y Sociedad, 24 (2) $167-177$

Sumario: 1. Hacia la erradicación del ácido en el grabado, 2. Del estudio a la adaptación de las técnicas de grabado en metal sin ácido, 3. De la electrólisis tradicional al sacrificio metálico, 4. Del grabado en hierro al cobre, cerrando el ciclo de los mordentes, 5.Barnices caseros, 6. Eliminando los solventes de petróleo, 7. Litografía con limón y miel en vez de ácidos inorgánicos, 8. Conclusión. Referencias.

\title{
1. Hacia la erradicación del ácido en el grabado
}

Como reseñó brevemente Chaves-Badilla (2010) en un artículo reciente en esta revista, las corrientes revolucionarias de una nueva modalidad de grabado libre del ácido nítrico, han calado fuerte en diversos países y la Escuela de Artes Plásticas, de la Universidad de Costa Rica (UCR), no es la excepción; por el contrario, somos un núcleo de investigación en grabado no tóxico, tratando de adaptar técnicas simples y seguras al entorno de un país en desarrollo y publicamos nuestras experiencias positivas, para allanar el camino a los nuevos artistas que desean acogerse a las modalidades no tóxicas del grabado.

En este artículo hacemos un sumario de nuestros hallazgos de un quinquenio trabajando sin ácido. Imaginamos que es posible que nuestro camino hacia la erradicación del ácido haya sido similar al de otros talleres; pues, la adopción de un nuevo proceso implica un cambio drástico del status quo, lo que usualmente enfrenta la resistencia a alejarse de una rutina dominada por el quehacer diario, para aventurarse en nuevos territorios con técnicas que había que estudiar y que ameritaban investigación para adaptarlas a las posibilidades del entorno de cada artista y sobre todo, porque cambiaban ligeramente la estética tradicional lograda con el cobre erosionado por el ácido nítrico. Dichosamente, las nuevas opciones de grabado han ido calando de forma gradual en el sentir de nuestros artistas y los vapores amarillentos del ácido nítrico en el taller y en las vías respiratorias, son solo un recuerdo del pasado que los nuevos estudiantes no han sufrido ${ }^{1}$ y que los 
grabadores tradicionales, incluyendo viejos profesores en otra época, aceptaron como parte de un proceso que no era cuestionado.

Afortunadamente las técnicas del grabado no tóxico han ido ganando adeptos día con día y las nuevas modalidades se impulsan con talleres y escritos, en los que se promueve tanto la técnica, como la bioseguridad; aplicada al artista, a su núcleo familiar e incluso al medio ambiente, lo que cala cada vez más fuerte gracias a una conciencia ecológica universal, que en nuestro contexto, prepara el terreno para la siembra del grabado no tóxico. Acorde con este pensamiento, en la Escuela de Artes Plásticas de la UCR impulsamos la publicación de las nuevas experiencias, pues cada día se presentan opciones para mejorar lo establecido por tradición y las soluciones son cada vez más simples y prácticas, como pretendemos mostrar en este artículo.

\section{Del estudio a la adaptación de las técnicas de grabado en metal sin ácido}

Las dos metodologías más difundidas del grabado sin ácido son la electrólisis y la electroquímica. En ambas, la electricidad es en última instancia la encargada de grabar la placa metálica; en la primera se aplica una fuente de corriente directa a un sistema, en el cual se cierra un circuito compuesto por dos placas metálicas sumergidas en un conductor líquido, denominado electrolito, que contiene iones del mismo metal a grabar; en un artículo previo (Hernández-Chavarría, Arias y Murillo, 2007a) hicimos una recopilación de conceptos al respecto, con las adaptación de los procesos a nuestras necesidades. En la segunda modalidad, se emplea el potencial eléctrico de los iones que conforman la solución mordente, en la cual, los iones reactivos roban electrones a la placa metálica, cuyos átomos ionizados entran en solución, por lo que se erosiona el metal y se realiza el hueco grabado (Hernández-Chavarría, Arias y Murillo, 2007b).

Nuestra experimentación en ambas modalidades nos ha llevado a hacer adaptaciones al entorno propio de la Escuela de Artes Plásticas, que ha llevado implícitamente la búsqueda de opciones cada vez más prácticas y económicas, para que sean sostenibles, aún para los estudiantes de escasos recursos económicos. Esto implica la búsqueda de materiales reciclables, incluyendo el propio metal y la preparación y uso de reactivos que puedan adquirirse en el comercio general, para obviar la importación de insumos; por ejemplo, una meta ha sido la confección de barnices con la mezcla de materiales de uso casero (Hernández-Chavarría, Murillo y Cambronero, 2009). Seguros de que nuestros aportes son de utilidad a grabadores de otras latitudes, a continuación hacemos un repaso de los cambios en los procesos, tratando de exponerlos de la manera más didáctica posible e instamos a los neófitos en la materia a consultar nuestros textos, que están disponibles en internet en la dirección del índice académico Redalyc, pues podría ayudarles a iniciarse en la aventura del grabado sin ácido.

\section{De la electrólisis tradicional al sacrificio metálico}

Desde el punto de vista técnico, una de las opciones más simples para grabado no tóxico es la electrólisis, pues la descripción del método y los principios físicos que le rigen son sencillos y aparte de ello, se requiere poco equipo; además, la idea de que 
el electrolito no se consume y se puede seguir usando por mucho tiempo, hace que el método sea muy tentador y que fácilmente gane adeptos.

La versión estándar de electrólisis parte del principio de utilizar una placa de metal para grabar, otra similar como receptora, ambas sumergidas en una solución que contenga iones del mismo metal, denominada electrolito, que en realidad es un conductor líquido para cerrar el circuito eléctrico formado por las dos placas, conectadas a una fuente de corriente directa. Con este sistema podemos fácilmente trabajar placas de cobre, hierro o aluminio, empleando como electrolitos soluciones de sulfato de cobre, cloruro ferroso o sulfato de aluminio (alumbre), respectivamente. En la figura 1 se muestra un esquema del equipo al que hacemos referencia.

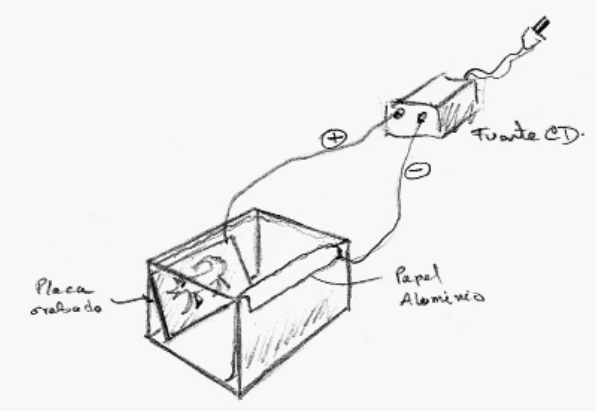

Figura 1. Esquema del equipo para electrólisis, empleando una placa de grabado, que puede ser de cobre, hierro o aluminio, según se use como electrolito sulfato de cobre, cloruro ferroso o alumbre, respectivamente, y como placa receptora se usa una hoja de papel de aluminio.

Analicemos algunas opciones que facilitan el proceso. Primero, la fuente de corriente directa, de preferencia un transformador que brinde entre 1 y 5 amperios (A), con un voltaje $(\mathrm{V})$ no mayor de $6 \mathrm{~V}$; sin embargo, podemos utilizar los transformadores de diversos equipos, incluyendo los cargadores de teléfonos móviles; aunque, muchos de estos equipos requieren muy poca corriente y usualmente brindan menos de un amperio; por ejemplo, la mayoría de los cargadores de teléfonos móviles requieren corrientes de 300 a $500 \mathrm{~mA}(0.3$ a $0.5 \mathrm{~A})$; estos se pueden utilizar, pero el tiempo requerido para grabar una placa es mucho mayor que el necesario si nuestra fuente brindara, por ejemplo 2A. El corolario es que no importa la potencia de nuestra fuente de corriente directa; lo importante es familiarizarse con ella, y la forma más simple es hacer una tira de pruebas al aguafuerte, para el metal que estemos utilizando; de manera similar a cómo se calcula el tiempo de exposición en el cuarto oscuro de fotografía. Para esto tomamos una tira de metal y la protegemos con barniz y hacemos unas rayas de unos $2 \mathrm{~cm}$ en un extremo; establecemos un intervalo de exposición a la corriente directa, por ejemplo, quince minutos, exponemos esa sección; posteriormente, rayamos el segmento siguiente y repetimos la exposición, continuando secuencialmente hasta que las rayas hayan recorrido toda la lámina. El resultado es una 
gradiente tonal acorde con la profundidad del grabado, según el tiempo de exposición a la corriente eléctrica, lo que se refleja en la cantidad de tinta que carga cada segmento de la gradiente. Con este ejercicio simple construimos una guía de trabajo; eso sí, hay que mantener fijas las condiciones empleadas: la misma la fuente de corriente directa y la misma cubeta de electrólisis, pues esta determina la distancia entre las dos placas. Ya que entre mayor sea esa distancia, mayor será la pérdida de corriente por resistencia en el sistema, que lo hace menos eficiente. En la figura 2 se muestra una tira de pruebas hecha en una lámina de aluminio con un cargador de $350 \mathrm{~mA}$.

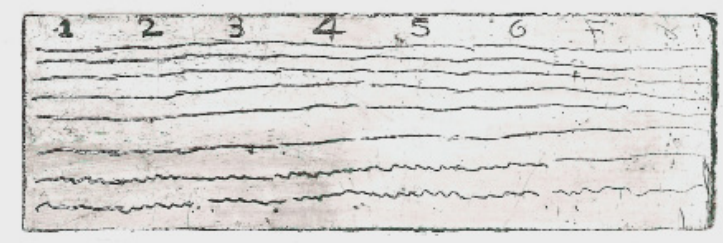

Figura 2. Tira de prueba en aluminio, sometida a 8 periodos de electrólisis, de 15 minutos cada uno, con un cargador de teléfono móvil de 350 miliamperios $(0.35 \mathrm{~A})$. La primera sección acumuló un total de 120 minutos y la número 8 solo 15 minutos.

Un segundo aspecto a considerar es la placa receptora, por lo general las descripciones del método, indican que el sistema se completa con dos placas del mismo metal, la que se va a grabar, que se conecta al polo positivo (ánodo) y la receptora al negativo (cátodo). La función de esa segunda placa es recibir los iones arrancados a la primera y por lo tanto, podemos sustituirla por otra placa de otro metal más barato. Por ejemplo, si estamos grabando una placa de cobre, podemos colocar en el electrodo negativo una pieza de aluminio, que puede ser desde una lámina de metal, hasta una hoja de papel aluminio, o bien, una plancha de litografía offset usada. El requisito fundamental es que la placa receptora sea al menos del mismo tamaño que la placa de grabado e idealmente más grande.

El sacrificio metálico es una versión más práctica de electrólisis. Históricamente fue la opción encontrada hace unos doscientos años para evitar la corrosión de las piezas de cobre de los cascos de los barcos, lo cual se logra adosándole unas piezas de cinc que se sacrifican, ya que el agua de mar las oxida en vez de atacar el cobre, debido a la diferente reactividad química de los dos metales. Su aplicación al grabado sigue el mismo principio y con este proceso grabamos metales menos nobles que el cobre, como lo son el hierro, el aluminio, el cinc e incluso el acero. Para esta opción de grabado montamos un equipo de electrólisis como el que describimos en la figura uno, solo que en el receptor, polo negativo, colocamos una lámina de cobre o bien, una maraña de alambres de este metal y en el polo positivo colocamos nuestra placa de grabado, que puede ser de hierro, aluminio, cinc o acero y, como electrolito para cualquiera de esos metales ponemos agua con sal de mesa, a razón de un cuarto de 
taza por cada litro de agua, esto es una concentración de sal mucho mayor que la del agua de mar. Como el nombre del método indica, el metal menos noble se sacrifica oxidándose, lo que equivale a erosionarse, que en este contexto equivale a grabarse (Hernández-Chavarría, 2010, 2011).

La ventaja de este método de electrólisis sobre el tradicional, es que utilizamos el mismo electrolito para grabar en hierro, aluminio, cinc e incluso acero inoxidable, y por estar constituido por simple sal de cocina, resulta en extremo barato y asequible; por otro lado, la fuente de corriente directa puede ser hasta el cargador de un teléfono y la cámara de electrólisis puede ser tan simple como un envase tetrabrick: ¡se acaban las escusas para no hacer grabado en metal!

\section{Del grabado en hierro al cobre, cerrando el ciclo de los mordentes}

El otro método de grabado, el electroquímico, emplea soluciones de sales que incluyen iones metálicos más reactivos que el metal de la placa de grabado y por lo tanto, la corroen; en el argot del grabado estas soluciones se denominan mordentes. Al final de cuentas, lo que ocurre entre el mordente y la placa metálica, es un robo de electrones, pues el ion activo del mordente los arranca del metal, erosionando su superficie, lo que equivale a grabar la placa.

Es común que usemos dos mordentes, uno para hierro, aluminio o cinc y otro para el cobre. Los disponemos en este orden para que el texto siguiente sea más claro. Veamos por qué: denominamos al primer mordente "Sulfato salino", este se prepara a partir de una solución de sulfato de cobre al 15\% (150g/litro de agua), lo que da una solución de color azul; luego, le vamos agregando cucharadas de sal de cocina y agitando hasta obtener un color verde esmeralda intenso, que se alcanza cuando en el fondo del recipiente aún queda un remanente de sal sin disolver; si la solución inicial de sulfato de cobre fuese menos concentrada, sería de un color celeste y al agregar sal se tornaría turquesa. Es preferible la concentración más alta (verde esmeralda) pues la reacción con el metal es más rápida, aunque variará según el tipo de metal, siendo el hierro el más duro de los tres. En aluminio la reacción es tan rápida que podemos darnos el lujo de hacer aguadas, tal como hacía Goya, solo que en nuestro caso, lo que aplicamos con un pincel es el mordente salino, para aumentar el valor tonal en una determinada zona de la placa. Pero, dejemos de lado el aluminio y sigamos con el orden propuesto inicialmente, esto es, que comenzamos trabajando en placas de hierro, pues la reacción química que ocurre durante el grabado, como mencionamos anteriormente, consiste en que el ión activo, en este caso cobre rodeado de iones de cloro, arranca electrones a los átomos de hierro, transformándolos en iones que entran en solución, dejando un hueco en la lámina de donde se desprendieron; entre tanto, los electrones arrancados, neutralizan los iones de cobre del mordente, que precipitan como átomos de cobre, esto es, el precipitado dorado rojizo que se va depositando sobre los surcos del grabado, por lo que deberíamos colocar la placa en posición vertical o boca abajo, para que ese cobre precipitado no interfiera con el proceso de grabado. Finalmente, después de varios grabados, nuestro mordente se habrá agotado y dejará de funcionar y su color habrá cambiado a un verde parduzco, pues químicamente es otro producto, y en este caso, se trata de cloruro ferroso. Y he aquí la magia 
alquímica de la química real, pues ese cloruro ferroso es el ingrediente inicial para hacer el mordente para grabar láminas de cobre; entonces, solo tenemos que oxidarlo un paso más, para transformarlo en cloruro férrico, el mordente buscado. Esa oxidación adicional la hacemos con agua oxigenada de 30 volúmenes o con el hipoclorito de calcio, comercializado como "cloro para piscinas" (Hernández-Chavarría, Lebi, Murillo y Aguilar, 2011). Anteriormente promulgábamos que el cloruro ferroso lo podíamos preparar disolviendo hierro en ácido muriático, $\mathrm{HCl}$ al 30\% (HernándezChavarría y Murillo, 2010); la nueva opción es menos peligrosa, pues obtenemos ese mismo producto como residuo al agotar el mordente de sulfato salino; o si se quiere, podemos poner una fuente de hierro, por ejemplo, clavos, directamente en el sulfato salino; obviamente, nosotros promulgamos el uso secuencial de grabado, primero en hierro y luego en cobre, para no desperdiciar reactivos.

Recapitulando la marcha de trabajo propuesta, se recomienda que durante un primer periodo se trabaje con hierro, grabándolo con sulfato salino y guardando todos los residuos de mordentes agotados en un recipiente plástico adecuado. Al cabo de un tiempo, se decanta el sobrenadante de los residuos con cuidado de no agitarlo, para no arrastrar el cobre metálico que se encuentra en el fondo. Ese líquido verdoso obtenido se oxida con cloro, como se explicó anteriormente, hasta que cambie a un color pardo muy opaco, que corresponde al mordente para grabar cobre. Pero aún hay más, los residuos del fondo del recipiente se pasan al taller de cerámica y allí se convierte en material para hacer esmaltes a base de cobre, logrando tonalidades azul verdosas, tan intensas como la cantidad de residuos que hayamos recuperado. Si estuviésemos trabajando inicialmente con aluminio, no vamos a poder recuperar otro mordente, pero, los residuos recuperados equivalen a materiales para esmaltes cerámicos, pues se trata de alúminas. Por lo tanto, nada se tiene que ir por el desagüe (HernándezChavarría et al., 2011)

\section{Barnices caseros}

La adquisición de un barniz protector para cubrir las placas metálicas puede estar tan cercana como un simple ¡clic!, tal como reza mucha de la publicidad que nos agobia a diario y las posibilidades suelen ser tan amplias como todo lo que se encuentra en la red. La oferta mundial incluye desde las formulaciones clásicas a base de asfalto, hasta productos más novedosos y amigables con la naturaleza. Pero, el artista grabador puede preparar sus propios barnices, hechos a base de materiales comunes, que se pueden adquirir en los comercios locales, como alternativa ante los productos de las casas comerciales especializadas. Entre las posibilidades que hemos encontrado figuran, desde la adaptación de las recetas tradicionales hasta nuevas opciones alternativas.

La fórmula más parecida a la receta clásica la hemos preparado empleando como fuente de asfalto la pasta comercial tapagoteras. Para ello, se diluye esta pasta con un solvente como espíritu mineral o varsol y se pasa a través de un colador para eliminar su componente de fibra sintética, se calienta en baño María y se le adiciona una porción fundida al calor de cera de abejas, equivalente a un 10\% (5g de cera por cada $50 \mathrm{ml}$ ). También, podemos sustituir la cera de abejas por crayolas de dibujo. En otra 
versión más simple, se sustituye el asfalto por cera en gel para pisos y se adiciona la cera de abejas o la crayola en la misma proporción $(10 \%)$ y se le da color con tinta litográfica u óleo negro para darle un acabado similar al obtenido con la versión clásica de barniz, o bien, se deja transparente para re-trabajar láminas para aguatinta. En ambos casos, el barniz se aplica sobre la placa metálica con un pincel, lo que se facilita calentando la placa previamente. Una vez que la placa se ha enfriado, el barniz se seca, dejando una superficie con un acabado ceroso ideal para trabajarla con una punta metálica (Hernández-Chavarría et al., 2009).

Otra posibilidad es sustituir el barniz tradicional por parafina, mezclada con cera de abejas o crayola; obviamente, debe aplicarse sobre una placa metálica lo suficientemente caliente para que funda la parafina, y una vez fría brinda una cubierta cerosa. Esta modalidad es útil para trabajar con mordentes salinos, pero no por electrólisis, pues la parafina tiende a desprenderse en las líneas del diseño durante el proceso electrolítico. También, podemos utilizar tempera a manera de barniz, con la ventaja de que es hidrosoluble, por lo que puede lavarse con agua, aunque algunas versiones de tempera contienen sustancias acrílicas que dificultan su lavado una vez secas, por lo que es importante ensayar las distintas posibilidades que ofrece el mercado; usualmente las temperas más baratas funcionan mejor para estos propósitos.

\section{Eliminando los solventes de petróleo}

Una de las preocupaciones que siempre debemos tener presente en los talleres de arte, es la exposición a sustancias nocivas, entre las cuales figuran los solventes derivados del petróleo (espíritu mineral, varsol, thinner, gasolina, etc.), que se tornan tan frecuentes que su presencia se asume como normal, por lo no se cuestiona su peligrosidad y son empleados indiscriminadamente, ya sea para limpiar utensilios y mesas de trabajo o incluso para quitarse la tinta de las manos. Pero, independientemente de lo familiar que sean en nuestro entorno de trabajo, debemos tener presente que se trata de sustancias nocivas, solo que los efectos adversos se presentan después de muchos años de exposición e incluyen desde problemas de piel crónicos, alteraciones cromosómicas, hasta daños al sistema nervioso central, leucemias y otros tipos de cáncer, como claman los investigadores biomédicos, pues la literatura científica es rica en reportes que documentan los efectos tóxicos de la exposición a estas sustancias, aún en el ambiente doméstico (Hooiveld, Haveman, Roskes, Bretveld, Burstyn y Roeleveld, 2006; Scélo, Metayer, Zhang, Wiemels, Aldrich, Selvin, Month, Smith y Buffler, 2009; Wang, Zhang, Lan, Holford, Leaderer, Zahm, Boyle, Dosemeci, Rothman, Zhu, Qin, Zheng, 2009; Berr, Vercambre, Bonenfant, Singh-Manoux, Zins, y Goldberg, 2010).

Podemos erradicar eso solventes del taller de artes plásticas, recurriendo a sustancias más amigables; por ejemplo, los aceites vegetales, como los de cocina, pueden emplearse satisfactoriamente para limpieza las tintas litográficas de superficies de trabajo, como mesas y rodillos e incluso de las manos, aunque, para este último propósito es más recomendable usar un aceite más delicado, como los diseñados para el cuidado de bebes, que aparte de su buen funcionamiento, no irritan la piel y tienen buen olor. Otra opción, es el limoneno, un extracto de cáscaras de cítricos, que brinda 
ese aroma característico a muchos productos alimenticios y cosméticos, que también se ha empleado como insecticida, repelente y plaguicida para controlar pulgas y garrapatas en mascotas domésticas, lo que señala indirectamente su bioseguridad (Sun, 2007). También se emplea para fabricar limpiadores desengrasantes domésticos e industriales, cuya efectividad es tal que han sustituido a sustancias tan fuertes como el xilol y muchos derivados del petróleo (Filipson, Bard, y Karlsson, 1998). Entonces, los limpiadores domésticos a base de limoneno constituyen una magnífica opción para la limpieza en el taller, sin embargo, debemos tener presente que es importante no exponer nuestra piel y mucosas a sustancias que pueden ser dañinas o irritantes y entre ellas podemos incluir a las tintas, independientemente de su naturaleza, por lo cual siempre es importante emplear guantes protectores, mascarillas y gafas de seguridad, además, de tener una buena ventilación para evitar la acumulación ambiental de vapores.

En nuestro taller hemos experimentado la preparación de un limpiador con ralladuras de cáscara de naranja o limón, suspendidas en alcohol de $95^{\circ}$, en una relación de 1:4. La extracción del limoneno se puede hacer pasivamente, dejando la mezcla en reposo unos dos a tres días, agitando de vez en cuando. Sin embargo, el procedimiento se puede acelerar irradiando a baja potencia la preparación en un horno de microondas; para ello se ajusta la intensidad del horno a un $10 \%$ y se irradia en periodos de uno a dos minutos. A esa potencia no se genera calor, pero se aceleran las moléculas facilitando su liberación, tal como se aceleran los procesos de fijación química en microscopia electrónica (Hernández-Chavarría y Guillén, 2000, Hernández-Chavarría y Vargas-Montero, 2001). Posteriormente se pasa la mezcla por un colador para eliminar los restos de ralladura del cítrico empleado; si fuese necesario concentrar el producto, se deja evaporar el alcohol dejando el frasco abierto, hasta que se reduzca el volumen a la mitad o menos. Este producto es útil para limpiar utensilios desde espátulas hasta mesas de trabajo.

\section{Litografía con limón y miel en vez de ácidos inorgánicos}

El último rescoldo del ácido nítrico que nos quedaba en el taller de grabado estaba presente en la técnica litográfica, junto con el ácido fosfórico, empleados respectivamente en los mordentes para la piedra y para las láminas de aluminio. Finalmente, también de aquí contamos con opciones para eliminarlo. Nuestra propuesta más novedosa y simple ha sido el diseño de un método litográfico, en el cual el mordente empleado se prepara mezclando partes iguales de jugo de limón con miel de abejas y para los grises más suaves se adiciona más miel, para diluir el ácido cítrico y hacer un mordente más suave, tal como describimos en detalle en un artículo publicado en la revista El Artista (Hernández-Chavarría, 2011)

\section{Conclusión}

Durante el último lustro, el taller de grabado de la Escuela de Artes Plásticas de la Universidad de Costa Rica, ha sido un laboratorio de experimentación en las técnicas del grabado no tóxico, afrontando holísticamente el reto de velar por la salud del artista en su nicho de trabajo, promoviendo una práctica segura en el taller, que 
implica no solo la sustitución de las sustancias nocivas por otras más amigables, sino también, el manejo ordenado y limpio del espacio del taller y la disposición a aceptar nuevos rasgos estéticos acordes con las nuevas metodologías e incluso a sacrificar la estética tradicional, en pos de la preservación de la salud sin afectar negativamente el medio, proponiendo el manejo adecuado de desechos y el reciclaje de residuos.

Parte de los frutos logrados, ha sido que los nuevos estudiantes que han ingresado durante este tiempo, se han incorporado naturalmente a las nuevas técnicas, aceptándolas sin prejuicios y con la mente abierta a la nueva sensibilidad estética, que brindan otros metales a parte del cobre, como matrices para grabado, atacándolos mediante electrólisis o con mordentes libres de los ácidos cáusticos y que incluso se pueden reciclar, lo que nos lleva a nuestra siguiente meta: El grabado sostenible.

\section{Referencias}

Berr, C., Vercambre, M. N., Bonenfant, S., Singh-Manoux, A., Zins, M. y Goldberg, M. (2010). Occupational exposure to solvents and cognitive performance in the Gazel Cohort: Preliminary results. Dement Geriatr Cogn Disord, 30(10), 12-19.

Chaves-Badilla, S.I. (2010). Hacia un huecograbado menos tóxico en la educación artística. Arte Individuo y Sociedad, 22 (1), 121-134.

Filipsson, A.F., Bard, J. y Karlsson, S. (1998). Concise International Chemical Assessment Document 5. Limonene. World Health Organization. Geneva, 5, 1-36.

Hernández-Chavarría F, Guillén, R. (2000). Microwave processing for scanning electron microscopy, European Journal of Morphology, 38(2), 109-111.

Hernández-Chavarría, F. y Vargas-Montero, M. (2001). Rapid contrasting of ultrathin sections using microwave irradiation with heat dissipation. Journal Microscopy, 203(2), 227-230.

Hernández-Chavarría, F., Arias, O. y Murillo, A. (2007a). De la alquimia al grabado metálico sin ácido: I. Una guía simple para el grabado electrolítico o anódico. El Artista 4, 25-35.

Hernández-Chavarría. F., Arias, O. y Murillo, A. (2007b). De la alquimia al grabado metálico sin ácido: II. Una guía simple para el grabado electroquímico. El Artista 4, 36-46.

Hernández-Chavarría, F., Murillo, A. y Cambronero, J. (2009). Grabado en metal: Trucos y consejos para el grabador contemporáneo. El Artista. 6, 89-101.

Hernández-Chavarría, F. y Murillo, A. (2010) Grabado en metal siguiendo las técnicas de grabado no tóxico. Revista Tecnología en Marcha, 23(3), 47-54.

Hernández-Chavarría, F. (2010). Sacrificio metálico: Agua salada y grabado en acero inoxidable, aluminio o hierro. ¡Nada más barato! El Artista, 7, 90-7.

Hernández-Chavarría, F. (2011). Aguafuerte en aluminio, hierro y acero con sal de mesa. Grabado y Edición, 6(27), 54-9.

Hernández-Chavarría, F., Lebi, E., Murillo, A. y Aguilar, C. (2011). Grabado en metal: La alquimia del reciclaje. El Artista, 8, 192-201.

Hernández-Chavarría, F. (2011). Con limón y miel: Una litografía alternativa, simple y rápida. El Artista, 8, 242-50.

Hooiveld, M., Haveman, W., Roskes, K., Bretveld, R., Burstyn, I. y Roeleveld, N. 
(2006). Adverse reproductive outcomes among male painters with occupational exposure to organic solvents. Occupped Environmenta Medicine, 63(10), 538544.

Scélo, G., Metayer, C., Zhang, L., Wiemels, J.L., Aldrich, M.C., Selvin, S., Month, S., Smith, M.T. y Buffler, P. (2009). Household Exposure to Paint and Petroleum Solvents, Chromosomal Translocations, and the Risk of Childhood Leukemia. Environmental Health Perspectives, 117(1), 133-139.

Sun, J. (2007). D-Limonene:Safety and Clinical Applications. Alternative Medicine. Review, 12(3), 259-264.

Wang, R., Zhang, Y., Lan, Q., Holford, T.R., Leaderer, B., Zahm, S.H., Boyle, P., Dosemeci, M., Rothman, N., Zhu, Y., Qin, Q., y Zheng, T.(2009).Occupational exposure to solvents and risk of non-Hodgkin lymphoma in Connecticut women. American Journal of Epidemiology, 169(2), 176-185.

\section{Notas}

1. La reacción del ácido nítrico $(\mathrm{HNO})$ con el cobre $(\mathrm{Cu})$ es: $3 \mathrm{Cu}+8 \mathrm{HNO} 3=3 \mathrm{Cu}(\mathrm{NO} 3) 2+4 \mathrm{H} 2 \mathrm{O}+2 \mathrm{NO}$ (óxido nitroso) A su vez el NO (gas incoloro) reacciona con el oxígeno atmosférico y forma NO2 (dióxido de nitrógeno). Este gas produce dolor de cabeza, náuseas, vómito, fatiga, mareos y afecta la respiración; a largo plazo los daños pulmonares son irreversibles 